\title{
A New Non-dominated Sorting Genetic Algorithm for Multi-Objective Optimization
}

\author{
Chih-Hao Lin and Pei-Ling Lin \\ Department of Information Management, Chung Yuan Christian University \\ Taiwan, R.O.C.
}

\section{Introduction}

Multi-objective optimization (MO) is a highly demanding research topic because many realworld optimization problems consist of contradictory criteria or objectives. Considering these competing objectives concurrently, a multi-objective optimization problem (MOP) can be formulated as finding the best possible solutions that satisfy these objectives under different tradeoff situations. A family of solutions in the feasible solution space forms a Pareto-optimal front, which describes the tradeoff among several contradictory objectives of an MOP. Generally, there are two goals in finding the Pareto-optimal front of a MOP: 1) to converge solutions as near as possible to the Pareto-optimal front; and 2) to distribute solutions as diverse as possible over the obtained non-dominated front. These two goals cause enormous search space in MOPs and let deterministic algorithms feel difficult to obtain the Pareto-optimal solutions. Therefore, satisfying these two goals simultaneously is a principal challenge for any algorithm to deal with MOPs (Dias \& Vasconcelos, 2002).

In recent years, several evolutionary algorithms (EAs) have been proposed to solve MOPs. For example, the strength Pareto evolutionary algorithm (SPEA) (Zitzler et al., 2000) and the revised non-dominated sorting genetic algorithm (NSGA-II) (Deb et al., 2002) are two most famous algorithms. Several extensions of genetic algorithms (GAs) for dealing with MOPs are also proposed, such as the niche Pareto genetic algorithm (NPGA) (Horn et al., 1994), the chaos-genetic algorithm (CGA) (Qi et al., 2006), and the real jumping gene genetic algorithm (RJGGA) (Ripon et al., 2007).

However, most existing GAs only evaluate each chromosome by its fitness value regardless of the schema structure, which is a gene pattern defined by fixing the values of specific gene loci within a chromosome. The schemata theorem proved by Goldberg in 1989 is a central result of GA's theory in which a larger of effective genomes implies a more efficient of searching ability for a GA (Goldberg, 1989).

Inspired by the outstanding literature of Kalyanmoy Deb, this study proposes an evaluative crossover operator to incorporate with the original NSGA-II. The proposed evaluative version of NSGA-II, named E-NSGA-II, can further enhance the advantages of the fast nondominated sorting and the diversity preservation of the NSGA-II for improving the quality of the Pareto-optimal solutions in MOPs. The proposed evaluative crossover imitates the gene-therapy process at the forefront of medicine and therefore integrates a new gene- 
evaluation method with a gene-therapy approach in the traditional uniform crossover scheme. The gene-evaluation method evaluates the merit of different genes between two mating parents by mutually exchanging these therapeutic genes one-by-one and observing the fitness variances. And then, the proposed evaluative crossover adopts a gene-therapy approach to cure the mating parents mutually with respect to their gene contribution to retain superior genomes in the evolutionary population.

The particular advantage of E-NSGA-II is that the gene-evaluation method can implicitly generate effective genome without explicitly analyzing the solution space by classical local search techniques. The performance of the proposed algorithm is experimented on nine unconstrained benchmark MOPs. The experiment results show that the E-NSGA-II not only can converge the nondominated solutions to the Pareto-optimal front but also can enhance the solution diversity to spread the achieved extent for all test MOPs.

The rest of this chapter is organized as follows. Section 2 introduces the genetic operators of the proposed E-NSGA-II. Section 3 describes numerical implementation and parameter setting. Section 4 reports the computational experiments on unconstrained MOPs and discusses the characteristics of E-NSGA-II. Finally, this chapter concludes with a summary in Section 5.

\section{Algorithm Description}

The NSGA proposed by Srinivas and Deb (1994) is one of the first EAs for MOPs (Srinivas \& Deb, 1994). The main idea of the NSGA is the ranking process executed before the selection operation. In 2002, Deb et al. proposed a revised version, named NSGA-II, by introducing fast non-dominated sorting and diversity preservation policies (Deb et al., 2002). Three features of NSGA-II are summarized as follows:

1) Computational complexity: NSGA-II uses a fast non-dominated sorting approach to substitute for the original sorting algorithm of NSGA in order to reduce its computational complexity from $\mathrm{O}\left(M P^{3}\right)$ to $\mathrm{O}\left(M P^{2}\right)$, where $M$ is the number of objectives and $P$ is the population size. This feature makes NSGA-II more efficient than NSGA for large population cases.

2) Elitism preservation: Replacement-with-elitism methods can monotonously enhance the solution quality and speed up the performance of GAs significantly (Ghomsheh et al., 2007). NSGA-II adopts $(\mu+\lambda)$-evolution strategy to keep elitism solutions and prevent the loss of good solutions once they are found. Successive population is produced by selecting $\mu$ better solutions from $\mu$ parents and $\lambda$ children.

3) Parameter reduction: Traditional sharing approach is a diversity ensuring mechanism that can get a wide variety of equivalent solutions in a population. However, a sharing parameter should be specified to set the sharing extent desired in a problem. Therefore, NSGA-II defines a density-estimation metric and applies the crowded-comparison operator with less required parameters to keep diversity between solutions.

In this study, the proposed E-NSGA-II stems from a concept different from traditional NSGA-II, particularly in terms of the gene-evaluation method. That is, the E-NSGA-II inherits the advantages of the NSGA-II and emphasizes the development of a new crossover operator and a modified replacement policy (Lin \& Chuang, 2007). 


\subsection{Generation of Initial Population}

A real coding representation is efficiently applied to solve numerical MOPs. Each test MOP is structured in the same manner and consists of $M$ objective functions (Deb, 1999):

$$
\begin{aligned}
& \text { Minimize } T(\vec{x})=\left(f_{1}(\vec{x}), \cdots, f_{M}(\vec{x})\right) \\
& \text { where } \quad \vec{x}=\left[x_{1}, x_{2}, \cdots, x_{n}\right]^{T} \text {. }
\end{aligned}
$$

Each decision variable is treated as a gene and encoded by a floating-point number. Each

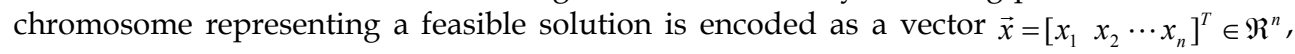
where $x_{i}$ denotes the value of the $i$ th gene and $n$ is the number of design variables in an MOP. Because the lower bound $\vec{l}=\left[\begin{array}{llll}l_{1} & l_{2} & \cdots & l_{n}\end{array}\right]^{T}$ and the upper bound $\vec{u}=\left[\begin{array}{llll}u_{1} & u_{2} & \cdots & u_{n}\end{array}\right]^{T}$ define the feasible solution space, the domain of each $x_{i}$ is denoted as interval $\left[l_{i}, u_{i}\right]$.

The main components of the E-NSGA-II are chromosome encoding, fitness function, selection, recombination and replacement. An initial population with $P$ chromosomes is randomly generated within the predefined feasible region. At each generation, E-NSGA-II applies the fast non-dominated sorting of NSGA-II to identify non-dominated solutions and construct the non-dominated front. And then, E-NSGA-II executes the rank comparison in selection operation to decide successive population by elitism strategy as the diversity preservation in NSGA-II (Deb et al., 2002). Therefore, the following sections only describe the details of the evaluative crossover operator and the diverse replacement.

\subsection{Evaluative Crossover}

For evaluation purpose, this study applies the crowding distance as an evaluation of chromosome's quality in the evaluative crossover. The crowding distance proposed in NSGA-II is used to estimate the density quantity of a particular solution in the population by calculating the average distance between other surrounding solutions with respect to each objective (Deb et al., 2002). After two parents have been selected from population, let the parent with larger crowding distance be named as the better parent $\left(\vec{x}_{b}\right)$ and the other one is the worse parent $\left(\vec{x}_{w}\right)$. Their crossover child is denoted as $\vec{y}$.

The proposed evaluative crossover imitates the gene-therapy process at the forefront of medicine, which inserts genes into an individual's cells to treat a disease by replacing a defective mutant allele by a functional one. Therefore, the evaluative crossover integrates a gene-evaluation method with a gene-therapy approach in the traditional uniform crossover scheme. By randomly generating a therapeutic mask with the same length as chromosomes, each parity bit in the therapeutic mask indicates whether the gene locus should be cured or not. For each gene locus, a random number in the interval $[0,1]$ is generated and compared to a pre-defined crossover rate $p_{c}$. If the random number is larger than the crossover rate, the parity bit in the therapeutic mask is assigned as 0 and no crossover occurs at this locus $\left(i \notin G_{c}\right)$. Otherwise, the parity bit in the therapeutic mask is assigned as 1 and the child's gene is generated by the gene-therapy approach $\left(i \in G_{c}\right)$.

Firstly, the gene-evaluation method mutually exchanges two parity genes between two mating parents and then compares their fitness variance as a measurement of these genes' merit. The exclusive features of the gene-evaluation method include that 1 ) the contribution 
of each gene is evaluated individually; and 2) the gene merit is estimated by the improvement of their density quantity during the gene-swap process (Lin \& He, 2007).

Secondly, one temporary chromosome is generated for crossover locus $i$, denoted as $\vec{t}_{i}=\left[x_{b 1}, \cdots, x_{b(i-1)}, \boldsymbol{x}_{w i}, x_{b(i+1)}, \cdots, x_{b n}\right]^{T}$. This temporary chromosome clones all alleles in the better parent and then replaces the selected gene of the better parent $\left(x_{b i}\right)$ with the one of the worse parent $\left(x_{w i}\right)$ in the same locus. The contribution of the gene $x_{w i}$ is denoted as $d_{w i}$ and approximated by the Euclidean distance between $\vec{t}_{i}$ and $\vec{x}_{w}$ by Equation (3). For comparison purpose, the Euclidean distance between $\vec{x}_{b}$ and $\vec{x}_{w}$ calculating by Equation (4) is the contribution of the gene $x_{b i}$ and denoted as $d_{b i}$. Therefore, comparing $d_{b i}$ with $d_{w i}$ can reveal the contributions of $x_{b i}$ and $x_{w i}$ with respect to the genetic material of the better parent.

$$
\begin{aligned}
& d_{w i}=\operatorname{dist}\left(\vec{t}_{i}, \vec{x}_{w}\right)=\sqrt{\sum_{m=1}^{M}\left(f_{m}\left(\vec{t}_{i}\right)-f_{m}\left(\vec{x}_{w}\right)\right)^{2}} \\
& d_{b i}=\operatorname{dist}\left(\vec{x}_{b}, \vec{x}_{w}\right)=\sqrt{\sum_{m=1}^{M}\left(f_{m}\left(\vec{x}_{b}\right)-f_{m}\left(\vec{x}_{w}\right)\right)^{2}}
\end{aligned}
$$

Finally, the gene-therapy approach can cure some defective genes in the better parent (i.e. $x_{b i}$ ) according to the genetic material of the other parent (i.e. $x_{w i}$ ) and then produce a child gene (i.e. $y_{i}$ ) for the evolutionary process. If the parity bit in the therapeutic mask is 0 (e.g. $i \notin G_{c}$ ), the offspring directly inherits the parity gene from the better parent, i.e. the gene in the better parent $\left(x_{b j}\right)$ is equal to that in the child (i.e. $y_{j}=x_{b j}$ ) at the same locus. On the other hand, if the parity bit in the therapeutic mask is 1 (e.g. $i \in G_{c}$ ), the therapy gene of the child at the same locus (i.e. $y_{j}$ ) is arithmetically combined from the parity genes of the mated parents (i.e. $x_{b j}$ and $x_{w j}$ ) according to their gene contributions. Each gene in the crossover child can be reproduced by Equation (5) in which coef is a random number in interval [0.5, 1.0].

$$
y_{i}= \begin{cases}x_{b i}, & \text { if }\left(i \notin G_{c}\right) \\ x_{b i} \times c o e f+x_{w i} \times(1-c o e f) & \text { if }\left(i \in G_{c}\right) \text { and }\left(d_{b i} \geq d_{w i}\right) \\ x_{b i} \times(1-c o e f)+x_{w i} \times c o e f, & \text { if }\left(i \in G_{c}\right) \text { and }\left(d_{b i}<d_{w i}\right)\end{cases}
$$

Example: In Fig. 1, the better parent with larger crowding distance (Cub_len $=0.8)$ is $P 1$ and the worse parent (Cub_len $=0.6$ ) is $P 2$. The therapy gene is the 2nd gene in chromosomes. The temporary chromosome $T$ clones all of genes in $P 1$ except for the 2 nd gene, which copies from $x_{22}$ in $P 2$. We assume that the Euclidean distance $\left(d_{12}\right)$ between $P 1$ and $P 2$ is 0.5 and the distance $\left(d_{22}\right)$ between $P 1$ and $T$ is 0.7 , which are used to estimate the gene contribution of $x_{12}$ and $x_{22}$, respectively. Because $d_{22}$ is larger than $d_{12}$, the 2nd gene in P2 $\left(x_{22}\right)$ is better than that in $P 1\left(x_{12}\right)$. Therefore, the child's gene $\left(x_{y 2}\right)$ inherits more genetic material from $x_{22}$ than $x_{12}$. The pseudo code of the evaluative crossover is described in Table 1.

\subsection{Polynomial Mutation}

Mutation operator is applied to enlarge the population diversity to escape from local optima and therefore enhance the exploration ability. E-NSGA-II inherits the polynomial mutation used by NSGA-II and operates as Equation (6) and (7) (Deb \& Goyal, 1996). 


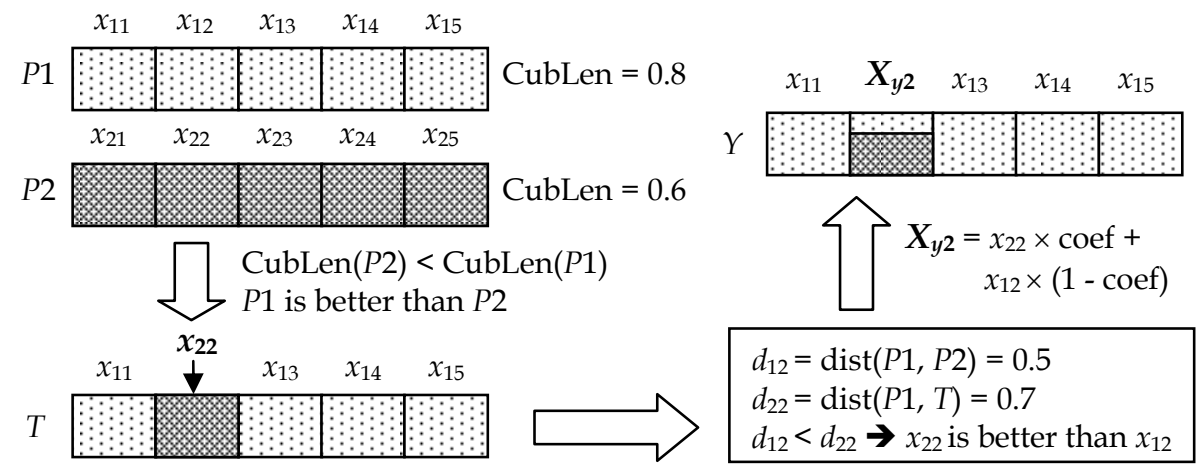

Fig. 1. An illustration of the gene-therapy approach

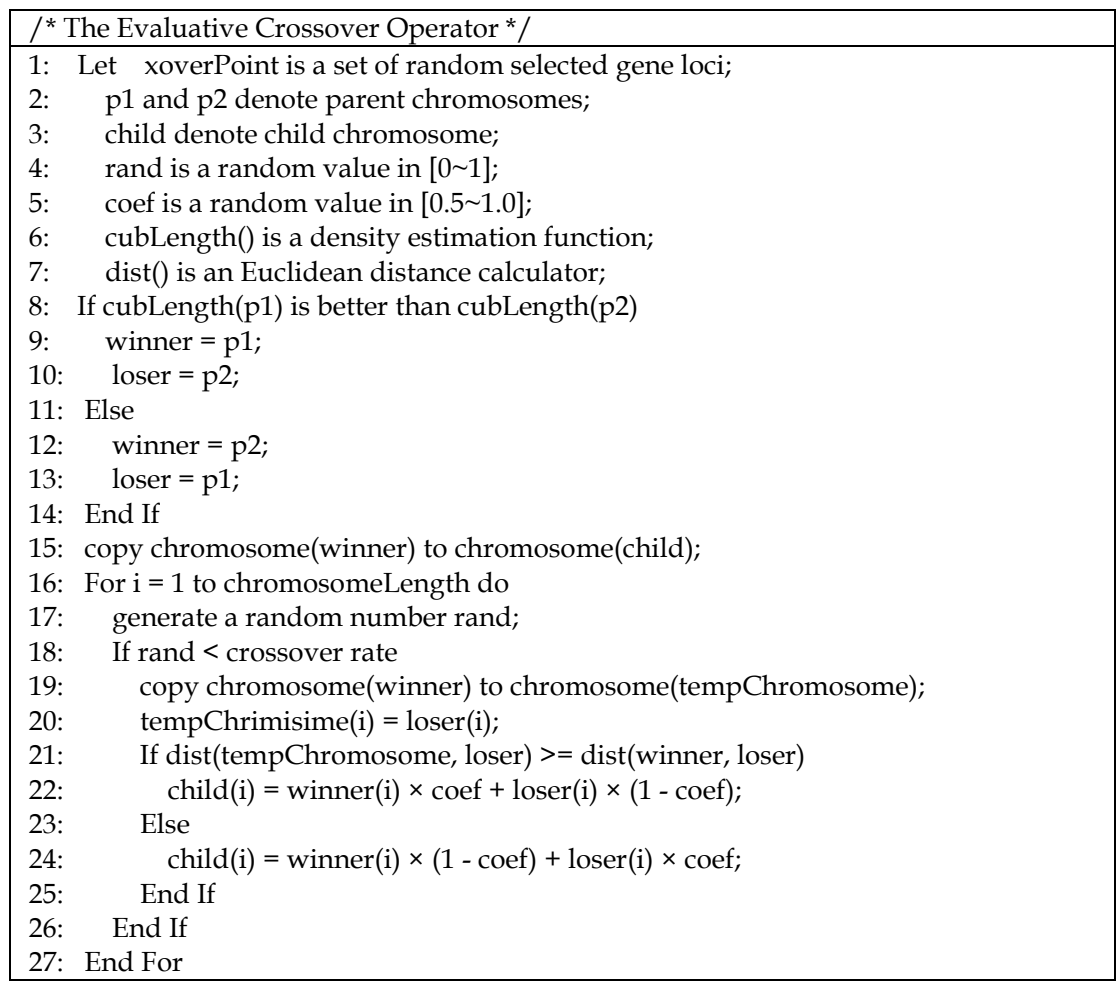

Table 1. The pseudo code of the evaluative crossover operator

$$
\begin{gathered}
y_{i}^{(1, t+1)}=x_{i}^{(1, t+1)}+\left(x_{i}^{(u)}-x_{i}^{(L)}\right) \overline{\delta_{i}} \\
\overline{\delta_{i}}=\left\{\begin{array}{ccc}
\left(2 r_{i}\right)^{1 /\left(\eta_{m}+1\right)}-1, & \text { if } & r_{i}<0.5 \\
1-\left[2\left(1-r_{i}\right)\right]^{1 /\left(\eta_{m}+1\right)}, & \text { if } & r_{i}<0.5
\end{array}\right.
\end{gathered}
$$


In Equation (6), $x_{i}^{(u)}$ and $x_{i}^{(L)}$ are the upper and lower bounds of the mutation parameter. According to Deb's research, the shape of the probability distribution is directly controlled by an external parameter $\eta_{m}$ and the distribution is not dynamically changed with generations (Deb, 2001). Therefore, parameter $\eta_{m}$ is also fixed in this study.

\subsection{Diverse Replacement}

E-NSGA-II modifies the replacement strategy proposed by Ghomsheh et al. in 2007 to keep diversity and generate successive population (Ghomsheh et al., 2007). The replacement criteria relying on the fast non-dominated sorting and diversity metric can keep those better diversity individuals and provide larger search space for crossover and mutation operators. In this study, a competitive population is generated by combining the parent population and the offspring population. In the competitive population, if the number of individuals with rank=1 is less than the population size, the successive population is firstly filled with the best non-dominated solutions and then selects the highest diversity solutions from the remaining individuals with rank $>1$ until the pre-specified population size is achieved. On the other hand, the successive population is sequentially filled with the best diversity solution from individuals with rank=1 until the size of the successive population is equal to the population size. According to these replacement criteria, the successive population can be generated. The pseudo code of replacement procedure is described in Table 2.

\section{Numerical Implementation}

For each test MOP, E-NSGA-II performs 10 runs with different seeds to observe the consistency of the outcome. The mean value of the measures reveals the average evolutionary performance of E-NSGA-II and represents the optimization results in comparison with other algorisms. The variance of the best solutions in 10 runs indicates the consistency of an algorithm. E-NSGA-II is implemented by MATLAB.

\subsection{Performance Measures}

Different performance measures for evaluating efficiency have been suggested in literature (Okabe et al., 2003). For comparison purpose, this study applies two metrics: 1) the convergence metric $(Y)$ : approximating the average distance to the Pareto-optimal front; and 2 ) the diversity metric $(\Delta)$ : measuring the extent of spread achieved among the obtained solutions (Deb, 2001).

For the convergence metric $(Y)$, a smaller metric value implies a better convergence toward the Pareto-optimal front. This study uses 500 uniformly spaced solutions to approximate the true Pareto-optimal. To measure the distance between the obtained non-dominated front $(Q)$ and the set of Pareto-optimal solutions $\left(P^{*}\right)$, this study computes the minimum Euclidean distance of each solution from 500 chosen points on the Pareto-optimal front by Equation (8). The average of these distances is used as the convergence metric as Equation (9).

$$
\begin{gathered}
d_{i}=\min _{k=1}^{\left|P^{*}\right|} \sqrt{\sum_{m=1}^{M}\left(f_{m}^{i}-f_{m}^{*(k)}\right)^{2}} \\
\mathrm{Y}=\left(\sum_{i=1}^{|Q|} d_{i}\right) /|Q|
\end{gathered}
$$




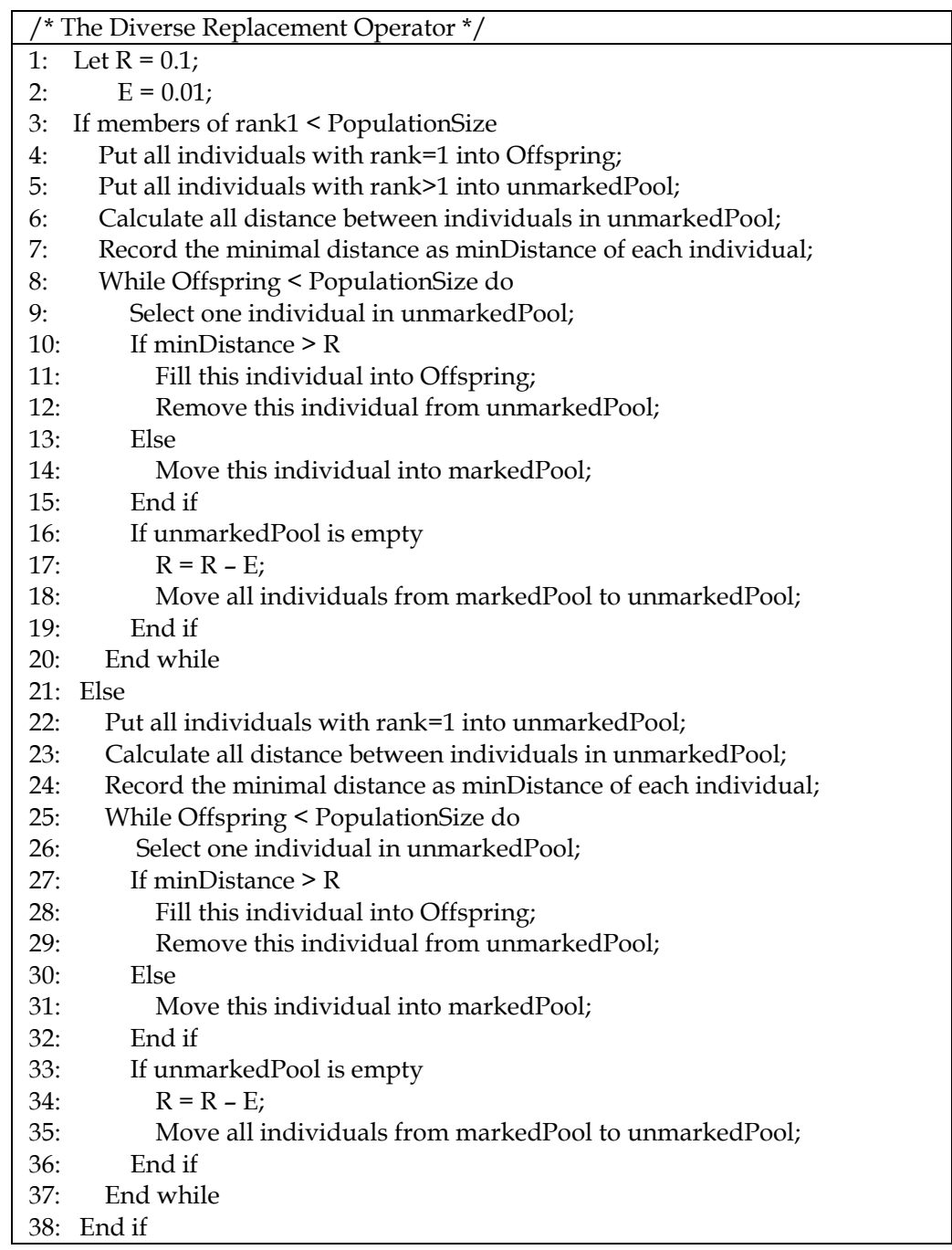

Table 2. The pseudo code of the diverse replacement operator

In Equation (8), $d_{i}$ is the Euclidean distance between the solution $i \in Q$ and the nearest member of $P^{*}$. Indicator $k$ denotes the $k^{\text {th }}$ member in $P^{*}$. Notation $M$ is the number of objectives and $f_{m}^{*(k)}$ is the $m^{\text {th }}$ objective function value of $k^{\text {th }}$ member in $P^{*}$. Indicator $i$ in Equation (9) is the obtained non-dominated solution from E-NSGA-II.

For Diversity metric $(\Delta)$, the value of $\Delta$ would be close to zero if the non-dominated solutions of the obtained front widely and uniformly spread out. The diversity metric $(\Delta)$ measures the extent of spread achieved among the obtained non-dominated solutions and is calculated by Equation (10). 


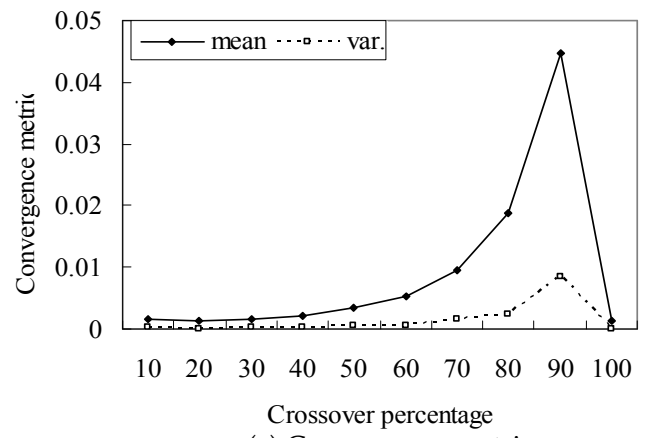

(a) Convergence metric

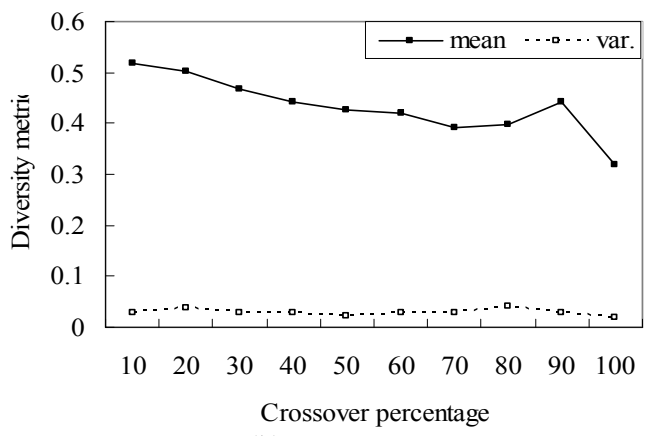

(b) Diversity metric

Fig. 2. Effect comparison among ten crossover percentages on problem ZDT1

$$
\Delta=\frac{d_{f}+d_{l}+\sum_{i=1}^{N-1}\left|d_{i}-\bar{d}\right|}{d_{f}+d_{l}+(N-1) \bar{d}}
$$

The Euclidean distances between the extreme solutions of the Pareto front $\left(P^{*}\right)$ is $d_{f}$. The distances between the boundary solutions of the obtained nondominated set $(Q)$ is $d_{l}$, and the distances between the consecutive solutions in the obtained non-dominated set is $d_{i}$. Notation $\bar{d}$ is the average of $d_{i}$.

\subsection{Parameter Setting}

To discover the best configuration for E-NSGA-II, some comprehensive investigations for parameter setting are performed on a benchmark problem. Especially, the performance of the evaluative crossover is influenced by three parameters: 1) crossover percentage; 2) crossover rate; and 3) therapeutic coefficient. The experimental results are averaged in 10 runs and evaluated by the convergence metric and the diversity metric. Problem ZDT1 is selected to analyze the effect of different parameters with a reasonable set of values in these experiments. The test function ZDT1 proposed by Zitzler et al. has a convex Pareto-optimal front and two objective functions without any constraint. The number of decision variables is 30 and the feasible region of each variable is in interval [0,1].

$$
\begin{aligned}
& \text { Minimize } \quad T(\vec{x})=\left(f_{1}\left(x_{1}\right), f_{2}(\vec{x})\right) \\
& \text { where } \\
& \qquad f_{1}\left(x_{1}\right)=x_{1} \\
& \quad f_{2}(\vec{x})=g(\vec{x})\left[1-\sqrt{x_{1} / g(\vec{x})} \mid\right. \\
& g(\vec{x})=1+9\left(\sum_{i=2}^{n} x_{i}\right) /(n-1) .
\end{aligned}
$$

\section{1) Effect of Crossover Percentage}

The crossover percentage decides how many successive individuals are produced by crossover operator. For example, $80 \%$ crossover percentage means that the crossover operator produces $80 \%$ offspring and the other $20 \%$ are produced by mutation operator. Especially, $100 \%$ crossover percentage means that all offspring are firstly recombined by crossover operator and then flipped one or more genes by mutation operator. 


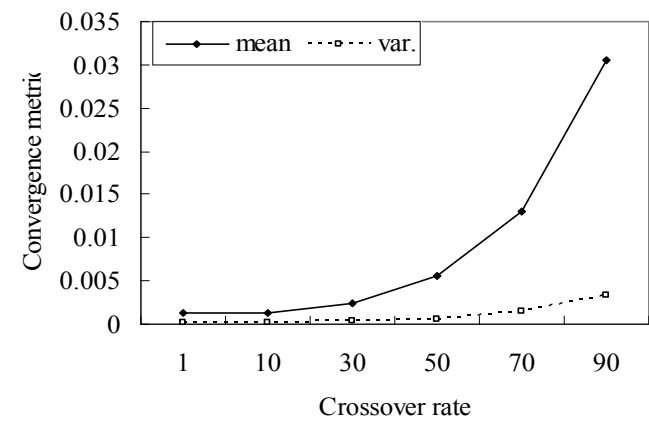

(a) Convergence metric

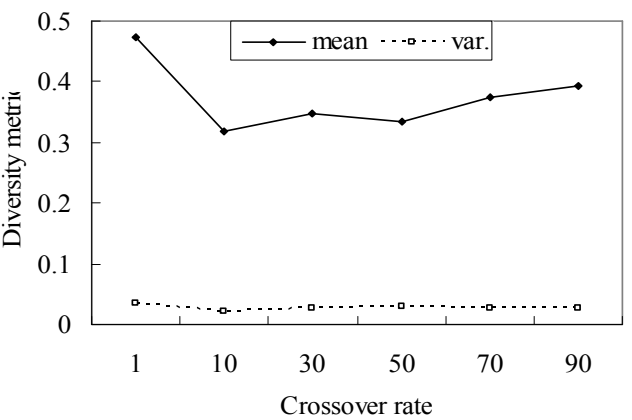

(b) Diversity metric

Fig. 3. Effect comparison among ten crossover rates on problem ZDT1

To analyze the best percentage of crossover children in each generation, ten crossover percentages (from 10\% to 100\%) were tested on problem ZDT1. The mean and variance of the convergence and diversity metrics are depicted in Fig. 2(a) and Fig. 2(b), respectively.

In Fig. 2(a), a larger crossover percentage implies a worse convergence situation when the crossover percentage is assigned from $10 \%$ to $90 \%$. However, the best convergence is obtained when the crossover percentage is $100 \%$. For diversity metric, Fig. 2(b) shows that the diversity metric is slightly declined from $10 \%$ to $70 \%$ and then rises until $90 \%$. In particular, the best diversity metric is also obtained when the crossover percentage is $100 \%$. Therefore, all individuals in this study are firstly recombined by the evaluative crossover and then mutated by the polynomial mutation.

\section{2) Effect of Crossover Rate}

In the gene-evaluation method, a smaller crossover rate implies a lower computational effort because only the selected loci in the therapeutic mask need to be evaluated individually. To realize the effects of different crossover rates $\left(p_{c}\right)$, six simulations with crossover rates from $1 \%$ to $90 \%$ are conducted on problem ZDT1 to discover the best crossover rate.

The convergence and diversity metrics of experimental results are depicted in Fig. 3(a) and $3(b)$, respectively. In Fig. 3(a), the convergence metric remains stable between $p_{c}=1 \%$ and $p_{c}=10 \%$. Obviously, a larger crossover rate implies a worse convergence situation on problem ZDT1 while $p_{c}$ is larger than $10 \%$. The diversity metric in Fig. 3(b) is monotonically decreased from $p_{c}=1 \%$ to $p_{c}=10 \%$ and then slightly increased until $p_{c}=90 \%$. Considering the convergence and diversity metrics, the crossover rate applied in this study is $10 \%$.

\section{3) Effect of Therapeutic Coefficient}

In the gene-therapy approach, each therapeutic gene of crossover child arithmetically combines two parity genes at the same locus of the mating parents. To test the effects of different therapeutic coefficient (coef), seven fixed coefficients and four variable ones for Equation (5) are tested on problem ZDT1. The mean and variance of the experimental results for seven fixed coefficients (from 0.01 to 1.0) are depicted in Fig. 4. The convergence metric depicted in Fig. 4(a) remains stable between 0.01 and 0.5. As the value of coef is larger than 0.5 , the convergence situation is dramatically increased until coef $=1$. When the value of coef increases from 0.01, the diversity metric in Fig. 4(b) is decreased and levels off between coef $=0.5$ and coef $=0.9$. And then, the diversity metric increases at coef $=1.0$. 


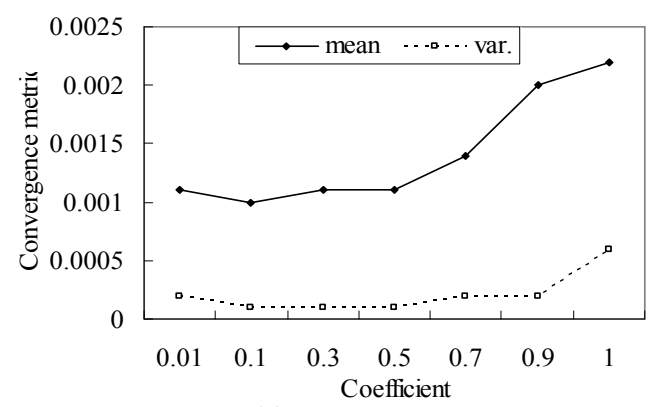

(a) Convergence metric

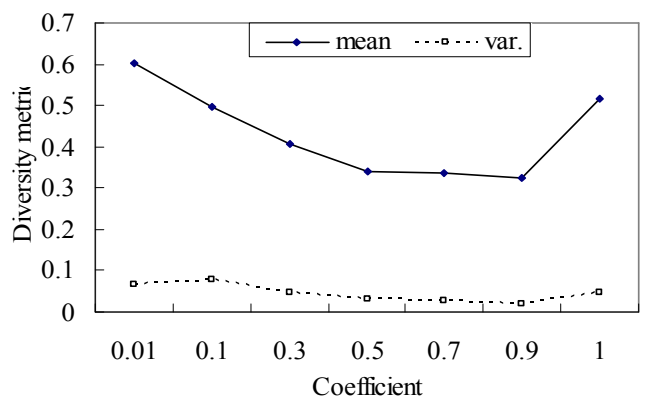

(b) Diversity metric

Fig. 4. Effect comparison among fixed therapeutic coefficients on problem ZDT1

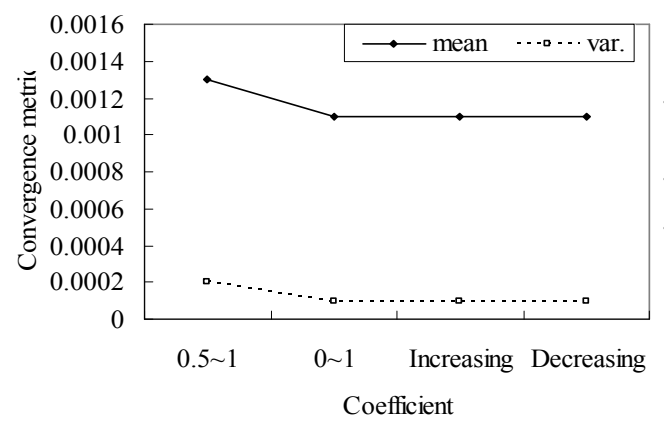

(a) Convergence metric

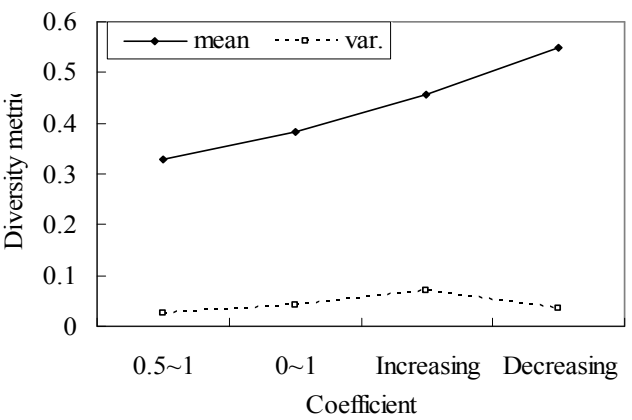

(b) Diversity metric

Fig. 5. Effect comparison among variable therapeutic coefficients on problem ZDT1

\begin{tabular}{|l|r|}
\hline \multicolumn{2}{|c|}{ E-NSGA-II Algorithm Setting } \\
\hline Population size & 100 \\
\hline Maximum generation & 1000 \\
\hline Simulation times & 10 \\
\hline Percentage of offspring reproduction from crossover operator & $100 \%$ \\
\hline Percentage of offspring reproduction from mutation operator & $100 \%$ \\
\hline \multicolumn{2}{|c|}{ E-NSGA-II Crossover Operator } \\
\hline Crossover rate & 0.1 \\
\hline Coefficient of arithmetical combination & Random [0.5,1] \\
\hline \multicolumn{2}{|c|}{ E-NSGA-II Mutation Operator } \\
\hline Mutation rate & $1 /$ length of variable \\
\hline Mutation scope & Rank *20 \\
\hline
\end{tabular}

Table 3. Parameter setting of E-NSGA-II

Fig. 5 depicts the experimental results of four variable type of therapeutic coefficients, which consist of 1) random value in interval $[0.5,1]$; 2) random value in interval $[0,1]$; 3) monotonically increasing value (from 0 to 1 ); and 4 ) monotonically decreasing value (from 1 to 0$)$. Obviously, the random coefficient in interval $[0.5,1]$ achieves the best diversity metric in Fig. 5(b) although its convergence result in Fig. 5(a) is slightly worse than others about 0.0002. Considering the tradeoff between the convergence and diversity metrics, this study suggests the random value in interval $[0.5,1]$ as the therapeutic coefficient in this study. 


\begin{tabular}{|c|c|c|c|c|}
\hline Prob. & $n$ & Bounds & Objective Functions & Comments \\
\hline $\mathrm{SCH}$ & 1 & $\left.-10^{3}, 10^{3}\right]$ & $f_{1}=x^{2} ; f_{2}=(x-2)^{2}$ & convex \\
\hline FON & 2 & {$[-4,4]$} & $f_{1}=1-\exp \left(-\sum_{i=1}^{3}\left(x_{i}-\frac{1}{\sqrt{3}}\right)^{2}\right) ; f_{1}=1-\exp \left(-\sum_{i=1}^{3}\left(x_{i}+\frac{1}{\sqrt{3}}\right)^{2}\right)$ & nonconvex \\
\hline POL & 2 & {$[-\pi, \pi]$} & $\begin{array}{l}f_{1}=\left[1+\left(A_{1}-B_{1}\right)^{2}+\left(A_{2}-B_{2}\right)^{2}\right] ; f_{2}=\left[\left(x_{1}+3\right)^{2}+\left(x_{2}+1\right)^{2}\right] \\
A_{1}=0.5 \sin 1-2 \cos 1+\sin 2-1.5 \cos 2 \\
A_{2}=1.5 \sin 1-\cos 1+2 \sin 2-0.5 \cos 2 \\
B_{1}=0.5 \sin x_{1}-2 \cos x_{1}+\sin x_{2}-1.5 \cos x_{2} \\
B_{2}=1.5 \sin x_{1}-\cos x_{1}+2 \sin x_{2}-0.5 \cos x_{2}\end{array}$ & $\begin{array}{l}\text { nonconvex, } \\
\text { disconnected }\end{array}$ \\
\hline KUR & 3 & {$[-5,5]$} & $\left.f_{1}=\sum_{i=1}^{n-1}\left(-10 \exp \left(-0.2 \sqrt{x_{i}^{2}+x_{i+1}^{2}}\right)\right) ; f_{2}=\sum_{i=1}^{n}\left(\left|x_{i}\right|^{0.8}+5 \sin x_{i}^{3}\right)\right)$ & nonconvex \\
\hline ZDT1 & 30 & {$[0,1]$} & $f_{1}=x_{1} ; f_{2}=g(x)\left[1-\sqrt{x_{1} / g(x)}\right] ; g(x)=1+9\left(\sum_{i=2}^{n} x_{i}\right) /(n-1)$ & convex \\
\hline ZDT2 & 30 & {$[0,1]$} & $f_{1}=x_{1} ; f_{2}=g(x)\left[1-\left(x_{1} / g(x)\right)^{2}\right], g(x)=1+9\left(\sum_{i=2}^{n} x_{i}\right) /(n-1)$ & nonconvex \\
\hline ZDT3 & 30 & {$[0,1]$} & $\begin{array}{l}f_{1}=x_{1} ; f_{2}=g(x)\left[1-\sqrt{x_{1} / g(x)}-\left(x_{1} / g(x)\right) \sin \left(10 \pi x_{1}\right)\right] \\
g(x)=1+9\left(\sum_{i=2}^{n} x_{i}\right) /(n-1)\end{array}$ & $\begin{array}{l}\text { convex, } \\
\text { disconnected }\end{array}$ \\
\hline ZDT4 & 10 & $\begin{array}{l}x_{1} \in[0,1] \\
x_{i} \in[-5,5] \\
i=2, \ldots, n\end{array}$ & $\begin{array}{l}f_{1}=x_{1} ; f_{2}=g(x)\left[1-\sqrt{x_{1} / g(x)}\right] \\
g(x)=1+10(n-1)+\sum_{i=2}^{n}\left[x_{i}^{2}-10 \cos \left(4 \pi x_{i}\right)\right]\end{array}$ & nonconvex \\
\hline ZDT6 & 10 & {$[0,1]$} & $\begin{array}{l}f_{1}=1-\exp \left(-4 x_{1}\right) \sin ^{6}\left(6 \pi x_{1}\right) ; \\
f_{2}=g(x)\left[1-\left(\frac{f_{1}(x)}{g(x)}\right)^{2}\right] ; g(x)=1+9\left[\frac{\sum_{i=2}^{n} x_{i}}{n-1}\right]^{0.25}\end{array}$ & $\begin{array}{l}\text { nonconvex, } \\
\text { nonuniformly } \\
\text { spaced }\end{array}$ \\
\hline
\end{tabular}

Table 4. Unconstrained test MOPs (All objectives are minimization functions)

\subsection{Configuration of the E-NSGA-II}

After these comprehensive experiments, the configuration and parameter setting of the proposed evaluative crossover are determined. Other parameters used in this study are the same as those in the original NSGA-II (Deb et al., 2002). The configuration of E-NSGA-II is summarized in Table 3 and used in the following section for performance comparison.

\section{Computational Experiments}

\subsection{Test Problems}

Nine test problems for MOPs are used in these experiments to systematically evaluate the performance of E-NSGA-II. These unconstrained benchmark problems suggested by Zitzler et al. cover a broad range of functionality characteristics with two objective functions (Zitzler et al., 2000). In this study, the test MOPs are denoted as SCH, FON, POL, KUR, ZDT1, ZDT2, ZDT3, ZDT4 and ZDT6. Table 4 describes the problem identifier, the number of variables, the feasible regions of decision variables, the function formulations, and the nature of the Pareto-optimal front for each problem (Deb et al., 2002). 


\subsection{Existing Algorithms for Comparison}

Several existing algorithms are applied for the entire test MOPs in literature. This study compares the results of five well-known algorithms with the proposed E-NSGA-II on nine test problems. These existing algorithms are:

1) NSGA-II (revised non-dominated sorting genetic algorithm) (Deb et al., 2002): By using the fast non-dominated sorting and diversity preservation, NSGA-II identifies nondominated solutions in the population and then executing the rank comparison in selection operation to decide successor population by elitism strategy.

2) n-NSGA-II (niching-NSGA-II) (Ghomsheh et al., 2007): n-NSGA-II modifies the elitism strategy of the NSGA-II according to the diversity value of each candidate individual. The purpose of this algorithm is to guarantee a better spread among the solutions.

3) SPEA (strength Pareto evolutionary algorithm) (Zitzler \& Thiele, 1999): By combining several features of previous multiobjective EAs in a unique manner, SPEA differs from several multi-criteria EAs in the kind of fitness assignment and the niching technique.

4) PAES (Pareto-archived evolution strategy) (Knowles \& Corne, 1999): PAES is a $(1+1)$ evolution strategy that comprises three parts: the candidate solution generator, the candidate solution acceptance function, and the Nondominated-Solutions archive. PAES represents the simplest approach to a multiobjective local search procedure.

5) MOTS (multi-objective Tabu search) (Jaeggi et al., 2004): Based on Tabu search, MOTS uses functional decomposition to perform parallel objective function evaluations at the H\&J local search and the diversification search and becomes a parallel multi-objective continuous Tabu search algorithm.

\subsection{Comparison Results among Algorithms}

Simulation results of the proposed E-NSGA-II on nine test problems are compared with five multi-objective optimizers, which are NSGA-II, n-NSGA-II, SPEA, PAES and MOTS. Table 5 and Table 6 depict the convergence metric $Y$ and the diversity metric $\Delta$ of the experimental results obtained using these six algorithms, respectively. The mean and variance of simulation results in 10 independent experiments are depicted in the first row and the second row of each algorithm in Table 5 and Table 6 . The mean of the metrics reveals the average evolutionary performance and represents the optimization results in comparison with other algorisms. The variance of the metrics indicates the consistency of an algorithm.

Table 5 shows that using the proposed evaluative crossover can further improve the convergence quality of NSGA-II on almost all problems except on problem POL. E-NSGA-II performs as good as n-NSGA-II to converge in six MOPs and outperforms n-NSGA-II in FON, POL and ZDT6. Furthermore, E-NSGA-II significantly overcomes SPEA, PAES and MOTS in eight problems but slightly loses on problem POL. In all cases with E-NSGA-II, the variance of convergence metric in ten runs is also small except in POL. That is, E-NSGA-II is great and consistent as n-NSGA-II and outperforms NSGA-II, SPEA, PAES and MOTS on the convergence capability.

In Table 6, E-NSGA-II outperforms all other algorithms dramatically on the mean of the diversity metric in almost all test problems except in POL and KUR with NSGA-II. That is, E-NSGA-II is a brilliant algorithm for MOPs to ensure a better spread among solutions and provide a good diversity although it slightly loses on the mean of convergence metric in two problems with NSGA-II. That is, E-NSGA-II can find a better spread of solutions than other algorithms on almost all test problems. 


\begin{tabular}{|l|c|c|c|c|c|c|c|c|c|c|}
\hline Algorithm & Y & SCH & FON & POL & KUR & ZDT1 & ZDT2 & ZDT3 & ZDT4 & ZDT6 \\
\hline \multirow{2}{*}{ E-NSGA-II } & Mean & 0.0033 & 0.0011 & 0.1143 & 0.0165 & 0.0013 & 0.0010 & 0.0046 & 0.0011 & 0.0042 \\
\cline { 2 - 10 } & Variance & 0.0002 & 0.0001 & 0.1008 & 0.0031 & 0.0001 & 0.0001 & 0.0003 & 0.0001 & 0.0002 \\
\hline \multirow{2}{*}{ n-NSGA-II } & Mean & 0.0032 & 0.0023 & 0.2375 & 0.0161 & 0.0011 & 0.0008 & 0.0042 & 0.0011 & 0.0139 \\
\cline { 2 - 10 } & Variance & 0.0002 & 0.0002 & 0.0428 & 0.0034 & 0.0001 & 0.0001 & 0.0003 & 0.0002 & 0.0018 \\
\hline \multirow{2}{*}{ NSGA-II } & Mean & 0.0034 & 0.0019 & 0.0156 & 0.0290 & 0.0335 & 0.0724 & 0.1145 & 0.5131 & 0.2966 \\
\cline { 2 - 10 } & Variance & 0.0000 & 0.0000 & 0.0000 & 0.0000 & 0.0048 & 0.0317 & 0.0079 & 0.1185 & 0.0131 \\
\hline \multirow{2}{*}{ SPEA } & Mean & 0.0034 & 0.1257 & 0.0378 & 0.0456 & 0.0018 & 0.0013 & 0.0475 & 7.3403 & 0.2211 \\
\cline { 2 - 10 } & Variance & 0.0000 & 0.0000 & 0.0001 & 0.0001 & 0.0000 & 0.0000 & 0.0000 & 6.5725 & 0.0005 \\
\hline \multirow{2}{*}{ PAES } & Mean & 0.0013 & 0.1513 & 0.0309 & 0.0573 & 0.0821 & 0.1263 & 0.0239 & 0.8548 & 0.0855 \\
\cline { 2 - 10 } & Variance & 0.0000 & 0.0009 & 0.0004 & 0.0119 & 0.0087 & 0.0369 & 0.0000 & 0.5272 & 0.0067 \\
\hline \multirow{2}{*}{ MOTS } & Mean & 0.0032 & 0.0008 & 0.0158 & 0.0276 & 0.0414 & 0.0664 & 0.0154 & 22.689 & 0.3758 \\
\cline { 2 - 10 } & Variance & 0.0000 & 0.0000 & 0.0005 & 0.0047 & 0.0008 & 0.0016 & 0.0010 & 10.966 & 0.1745 \\
\hline
\end{tabular}

Table 5. Mean (first rows) and variance (second rows) of the convergence metric $\Upsilon$

\begin{tabular}{|l|c|c|c|c|c|c|c|c|c|c|}
\hline Algorithm & $\Delta$ & SCH & FON & POL & KUR & ZDT1 & ZDT2 & ZDT3 & ZDT4 & ZDT6 \\
\hline \multirow{2}{*}{ E-NSGA-II } & Mean & 0.2069 & 0.1629 & 0.9197 & 0.5771 & 0.1251 & 0.1311 & 0.5833 & 0.2001 & 0.1177 \\
\cline { 2 - 10 } & Variance & 0.0115 & 0.0125 & 0.0393 & 0.0377 & 0.0013 & 0.0093 & 0.0242 & 0.0201 & 0.0098 \\
\hline \multirow{2}{*}{ n-NSGA-II } & Mean & 0.3822 & 0.3590 & 0.9531 & 0.5004 & 0.4225 & 0.4238 & 0.6827 & 0.4623 & 0.4056 \\
\cline { 2 - 11 } & Variance & 0.0409 & 0.0270 & 0.0703 & 0.0404 & 0.0218 & 0.0368 & 0.0208 & 0.0371 & 0.0376 \\
\hline \multirow{2}{*}{ NSGA-II } & Mean & 0.4779 & 0.3781 & 0.4522 & 0.4115 & 0.3903 & 0.4308 & 0.7385 & 0.7026 & 0.6680 \\
\cline { 2 - 10 } & Variance & 0.0035 & 0.0006 & 0.0029 & 0.0010 & 0.0019 & 0.0047 & 0.0197 & 0.0646 & 0.0099 \\
\hline \multirow{2}{*}{ SPEA } & Mean & 1.0211 & 0.7924 & 0.9727 & 0.8530 & 0.7845 & 0.7551 & 0.6729 & 0.7985 & 0.8494 \\
\cline { 2 - 10 } & Variance & 0.0044 & 0.0055 & 0.0085 & 0.0026 & 0.0044 & 0.0045 & 0.0036 & 0.0146 & 0.0027 \\
\hline \multirow{2}{*}{ PAES } & Mean & 1.0633 & 1.1625 & 1.0200 & 1.0798 & 1.2298 & 1.1659 & 0.7899 & 0.8705 & 1.1531 \\
\cline { 2 - 10 } & Variance & 0.0029 & 0.0089 & 0.0000 & 0.0138 & 0.0048 & 0.0077 & 0.0017 & 0.1014 & 0.0039 \\
\hline \multirow{2}{*}{ MOTS } & Mean & 0.3789 & 1.1298 & 1.5933 & 1.1166 & 0.7714 & 0.8135 & 0.8234 & 1.0044 & 0.3722 \\
\cline { 2 - 10 } & Variance & 0.0251 & 0.0344 & 0.0120 & 0.1437 & 0.0789 & 0.0360 & 0.0225 & 0.0047 & 0.2648 \\
\hline
\end{tabular}

Table 6. Mean (first rows) and variance (second rows) of the diversity metric $\Delta$

\subsection{Algorithm Analysis}

According to the research by Huband et al. in 2006, each test problem can be characterized by four factors (Huband et al., 2006): 1) uni-modal/multi-modal, 2) convex/non-convex, 3) connected/disconnected, and 4) bias/non-bias. The modality of a test problem can determine the exploration ability of an algorithm for finding global optima. The geometric shape of the Pareto-optimal front can measure the selection and ranking ability of algorithms. The bias factor directly influences the convergence speed toward the Paretooptimal front of algorithms. If the test problem has several disconnected Pareto-optimal sets, algorithms will feel difficult to find all regions of the Pareto optimal front. The characters of nine test problems are depicted in Table 7 and analyzed in the follows.

\section{1) Multi-modal Problems}

A multimodal function possesses numerous local optima that could trap an algorithm into its local optima and fail to find global optima. In general, solving multimodal problems is more difficult than unimodal ones. In this study, problem POL, KUR, ZDT3, ZDT4 and ZDT6 contain mutimodal objective functions. 


\begin{tabular}{|c|c|c|c|c|c|}
\hline Problem & Objective & Modality & Convexity & Bias & Connectivity \\
\hline $\mathrm{SCH}$ & $\begin{array}{l}\text { f1 } \\
\text { f2 }\end{array}$ & $\begin{array}{l}\text { Uni-modal } \\
\text { Uni-modal }\end{array}$ & Convex & Non-bias & Connected \\
\hline FON & $\begin{array}{l}\text { f1 } \\
\text { f2 }\end{array}$ & $\begin{array}{l}\text { Uni-modal } \\
\text { Uni-modal }\end{array}$ & Non-convex & Non-bias & Connected \\
\hline POL & $\begin{array}{l}\text { f1 } \\
\text { f2 }\end{array}$ & $\begin{array}{l}\text { Multi-modal } \\
\text { Uni-modal }\end{array}$ & Non-convex & Non-bias & Disconnected \\
\hline KUR & $\begin{array}{l}\text { f1 } \\
\text { f2 }\end{array}$ & $\begin{array}{l}\text { Uni-modal } \\
\text { Multi-modal }\end{array}$ & Non-convex & Non-bias & Disconnected \\
\hline ZDT1 & $\begin{array}{l}\text { f1 } \\
\text { f2 }\end{array}$ & $\begin{array}{l}\text { Uni-modal } \\
\text { Uni-modal }\end{array}$ & Convex & Non-bias & Connected \\
\hline ZDT2 & $\begin{array}{l}\text { f1 } \\
\text { f2 }\end{array}$ & $\begin{array}{l}\text { Uni-modal } \\
\text { Uni-modal }\end{array}$ & Non-convex & Non-bias & Connected \\
\hline ZDT3 & $\begin{array}{l}\text { f1 } \\
\text { f2 }\end{array}$ & $\begin{array}{c}\text { Uni-modal } \\
\text { Multi-modal }\end{array}$ & Convex & Non-bias & Disconnected \\
\hline ZDT4 & $\begin{array}{l}\text { f1 } \\
\text { f2 }\end{array}$ & $\begin{array}{l}\text { Uni-modal } \\
\text { Multi-modal }\end{array}$ & Non-convex & Non-bias & Connected \\
\hline ZDT6 & $\begin{array}{l}\mathrm{f} 1 \\
\mathrm{f} 2\end{array}$ & $\begin{array}{l}\text { Multi-modal } \\
\text { Multi-modal }\end{array}$ & Non-convex & Bias & Connected \\
\hline
\end{tabular}

Table 7. Characters of nine test problems

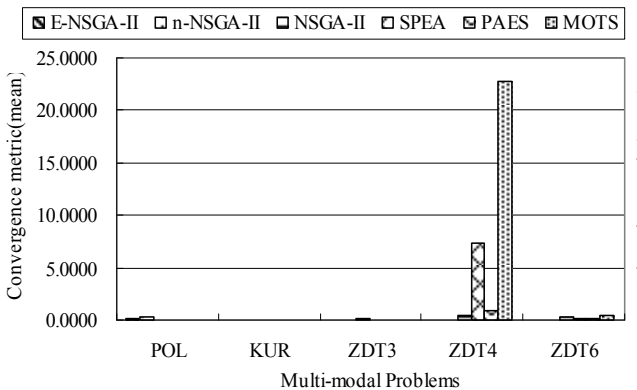

(a) Convergence metric

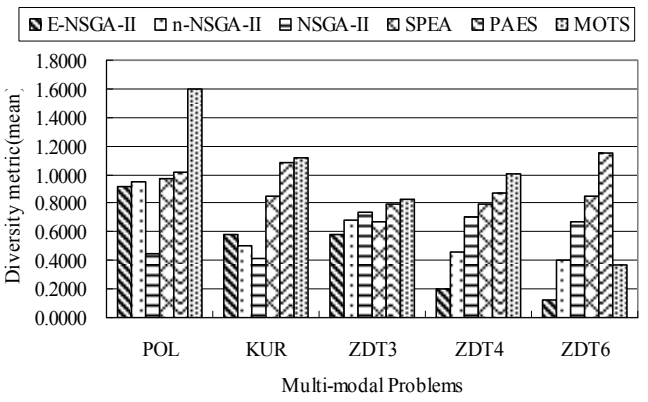

(b) Diversity metric

Fig. 6. Comparison between E-NSGA-II and existing algorithms on multimodal problems

In Fig. 6(a), the proposed E-NSGA-II can converge in these five multimodal problems as better as n-NSGA-II. Nevertheless, E-NSGA-II overcomes considerably NSGA-II, SPEA, PAES and MOTS on KUR, ZDT3, ZDT4 and ZDT6. In Fig. 6(b), E-NSGA-II outperforms all other algorithms on the mean of the diversity metric in almost all test problems except in POL and KUR with NSGA-II.

\section{2) Convex Problems}

Convex Pareto optimal fronts can cause difficulty for algorithms to rank solutions by the number of their dominated solutions because solutions around the middle of the convex Pareto front have great chance to dominate more solutions (Deb, 1999). Problem SCH, ZDT1 and ZDT3 belong to convex problems. The convergence metric $Y$ and the diversity metric $\Delta$ of the experimental results obtained using these six algorithms are depicted in Fig. 7(a) and Fig. 7(b), respectively. 


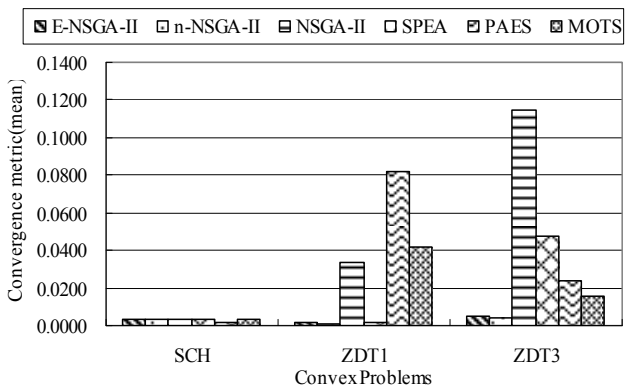

(a) Convergence metric

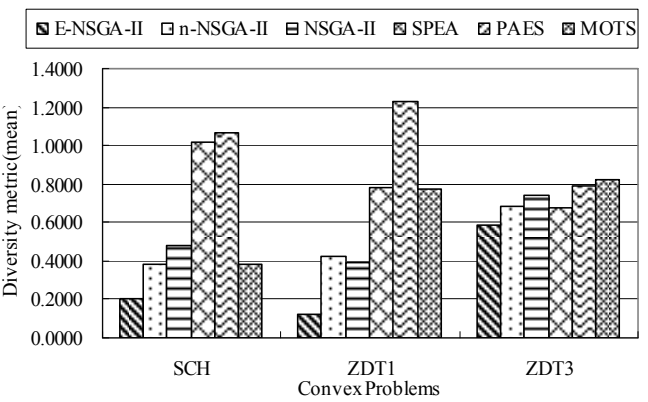

(b) Diversity metric

Fig. 7. Comparison between E-NSGA-II and existing algorithms on convex problems

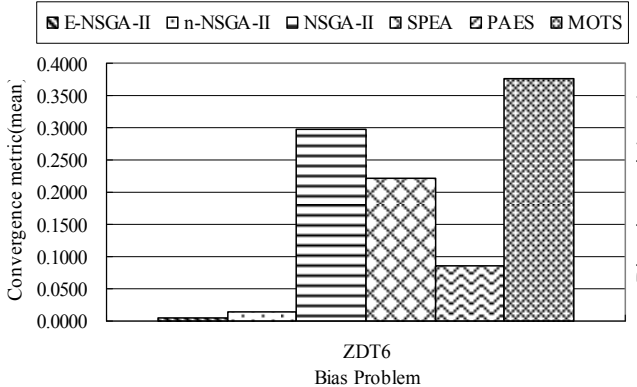

(a) Convergence metric

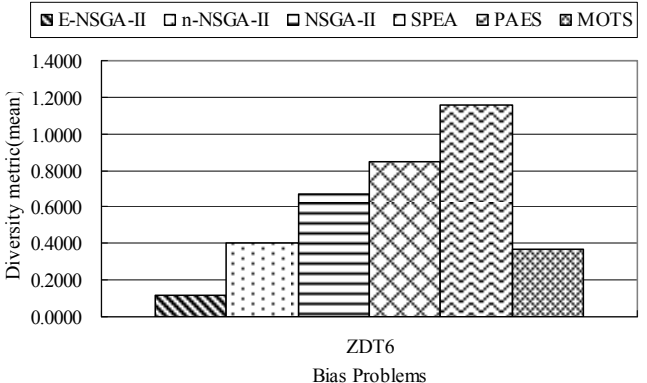

(b) Diversity metric

Fig. 8. Comparison between E-NSGA-II and existing algorithms on bias problem

Although dealing with the convex problems is difficult, the proposed E-NSGA-II performs the best convergence metric in Fig. 7(a) and the best diversity metric in Fig. 7(b) on all convex problems than other five algorithms. That is, the evaluative crossover can further improve the ranking performance of NSGA-II for convex problems.

\section{3) Bias Problem}

A bias problem may directly influences the convergence speed toward the Pareto-optimal front of algorithms. A better exploitation ability of an algorithm is useful to be able to identify the presence of bias in a test problem. In this study, only problem ZDT6 belongs to the bias problem. In Fig. 8(a) and Fig. 8(b), the convergence metric $(Y)$ and the diversity metric $(\Delta)$ show that E-NSGA-II with the proposed evaluative crossover is the best evolutionary algorithm among these six algorithms for handling the bias problem ZDT6.

\section{4) Disconnected Problems}

In this study, problem POL, KUR and ZDT3 have disconnected Pareto-optimal fronts, which will increase the likelihood that an algorithm will fail to find all regions of the Pareto optimal front. For KUR and ZDT3 in Fig. 9(a), the proposed E-NSGA-II can converge on the Pareto-optimal front and achieve a better convergence metric than other algorithms. Furthermore, E-NSGA-II also can spread solutions around and outperform other four algorithms (except for NSGA-II on POL and KUR) on the diversity metric in Fig. 9(b). 


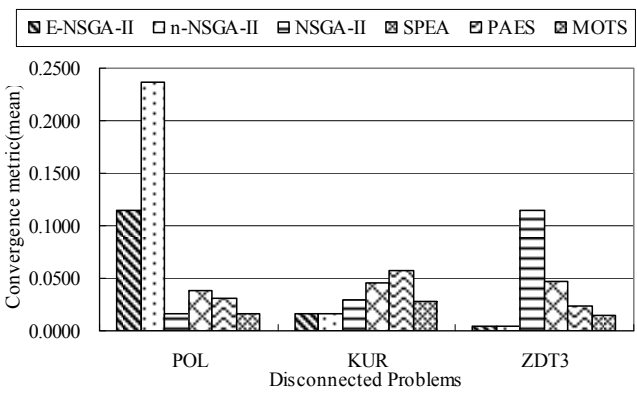

(a) Convergence metric

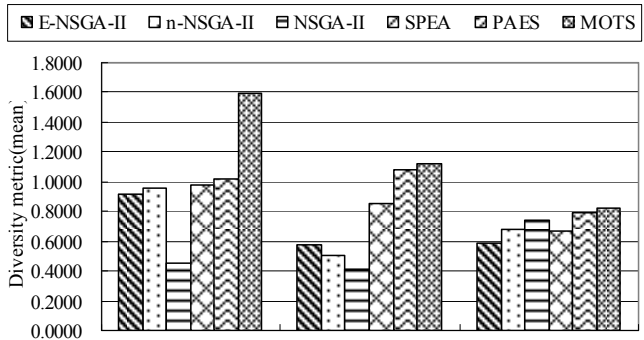

POL ZDT3

(b) Diversity metric

Fig. 9. Comparison between E-NSGA-II and existing algorithms on disconnected problems

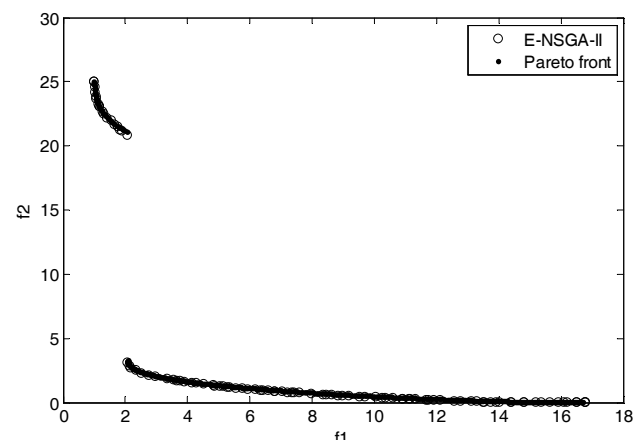

(a) POL

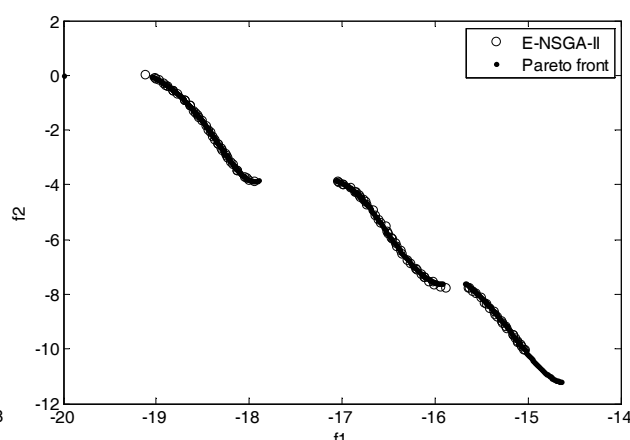

(b) KUR

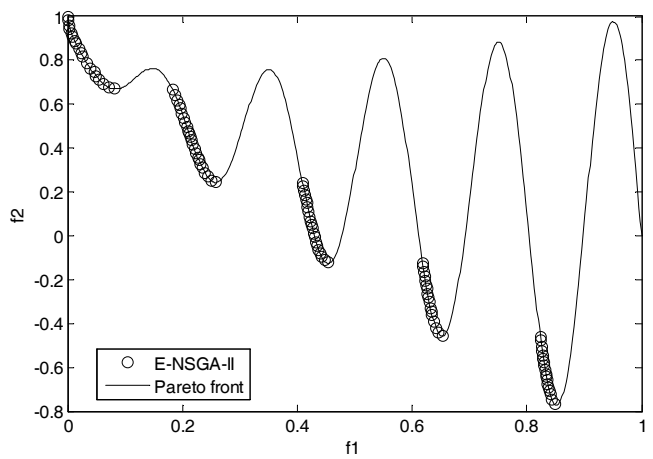

(c) ZDT3

Fig. 10. Nondominated solutions with E-NSGA-II on three disconnected test problems

For three disconnected problems, Fig. 10 shows all nondominated solutions obtained by using E-NSGA-II and the Pareto-optimal region. In their Pareto-optimal front, problem POL, KUR and ZDT3 have two, three and five regions of discontinuous curves, respectively. Fig. 10 demonstrates the ability of E-NSGA-II to converge the true Pareto-optimal front and spread diverse solutions in the front. Fig. 11 depict the Pareto-optimal front and nondominated solutions obtained by E-NSGA-II for the other six test problems where solid line 


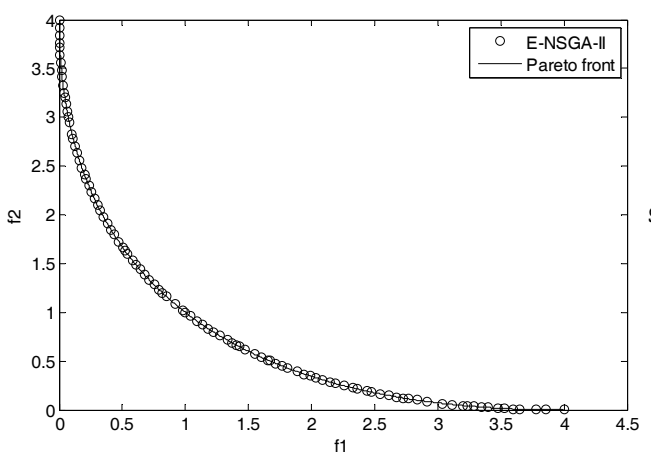

(a) $\mathrm{SCH}$

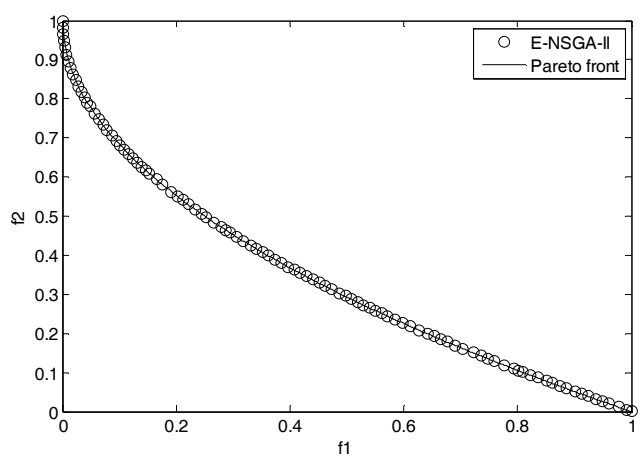

(c) ZDT1

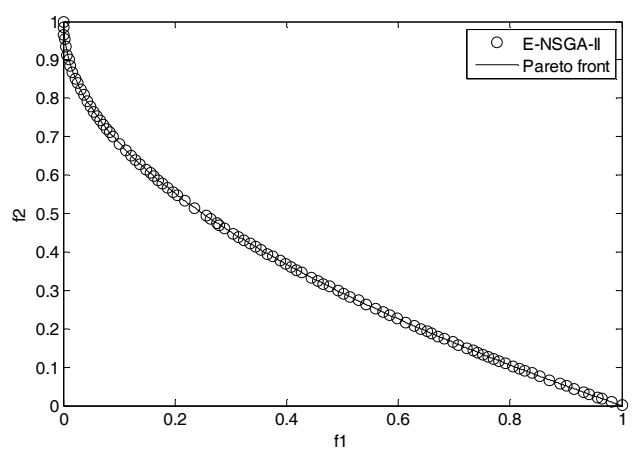

(e) ZDT4

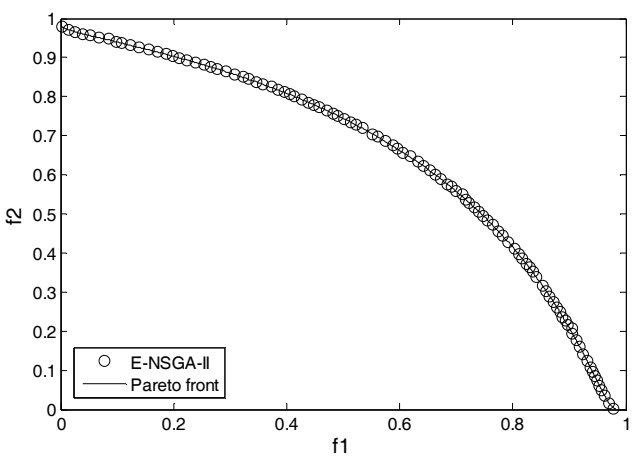

(b) FON

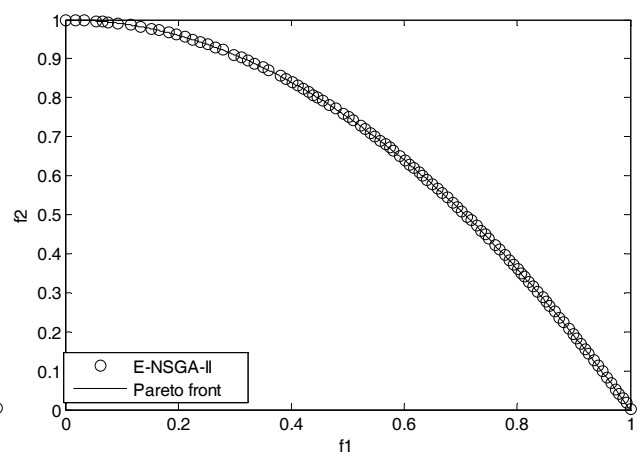

(d) ZDT2

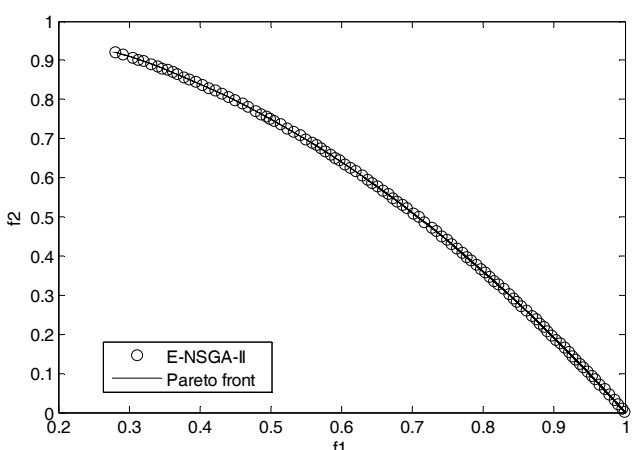

(f) ZDT6

Fig. 11. Nondominated solutions with E-NSGA-II on six test problems

represents the Pareto-optimal front and the hollow circles represent the obtained nondominated solutions with E-NSGA-II. Obviously, the solutions obtained by E-NSGA-II are very close to Pareto-optimal front and the spreading diversity is also excellent. Therefore, the E-NSGA-II is an effective GAs for solving MOPs and achieving excellent diversity metric. 


\section{Conclusion}

This study imitates the gene-therapy process at the forefront of medicine and proposes an innovative evaluative crossover operator. The evaluative crossover integrates a geneevaluation method with a gene-therapy approach in the traditional NSGA-II for finding uniformly distributed Pareto-optimal front of multi-objective optimization problems. To further enhance the advantages of fast non-dominate sorting and diversity preservation in NSGA-II, the proposed gene-evaluation method partially evaluates the merit of different crossover genes by substituting them in better parent and then calculating the fitness variances. The gene-therapy approach incorporates with the evaluative crossover to cure the mating parents mutually with respect to their gene contribution in order to retain superior genomes in the evolutionary population.

Some comprehensive investigations for parameter setting are performed on a benchmark problem. Especially, three parameters of the evaluative crossover with a reasonable set of values are analyzed to realize their evolutionary effect. The experimental results show that a $100 \%$ crossover percentage with $10 \%$ crossover rate and a random therapeutic coefficient can achieve the best performance for E-NSGA-II.

The proposed algorithm is tested on nine unconstrained multi-objective optimization problems. The experimental results are compared with five existing algorithms. The results show that the proposed E-NSGA-II is able to converge the Pareto-optimal front of all test problems, even though other algorithms experiences difficulties in approaching the global optima on some functions. E-NSGA-II can also achieve better diversity qualities than others. The results of algorithm analysis also reveal that the proposed evaluative crossover can intuitively evaluate gene contributions that can guide E-NSGA-II to perform an efficient search by dynamically shifting emphasis to significant genome in the feasible space without abdicating any portion of the candidate schemata. Although the convergence metric on the disconnected problem POL is slightly worse than four algorithms, E-NSGA-II outperforms almost all other algorithms on the mean of the convergence metric in other test problems. For the diversity metric, E-NSGA-II also performs better than all other algorithms dramatically in all test problems except for NSGA-II on POL and KUR.

In the future, the proposed E-NSGA-II should further develop the properties of a simple yet efficient evaluative crossover operator, a revised mutation operator and a parameter-less approach to deal with a wide spectrum of real world multi-objective problems.

\section{Acknowledgement}

The authors would like to thank Kalyanmoy Deb for his explicit writing and brilliant papers that inspire our mind. The research is supported by the National Science Council of Republic of China under grant NSC 96-2221-E-033-007.

\section{References}

Deb, K. (1999). Multi-objective genetic algorithms: Problem difficulties and construction of test problems. Evolutionary Computation, Vol. 7, No. 3, pp. 205-230.

Deb, K. (2001). Multi-objective optimization using evolutionary algorithms, New York: John Wiley and Sons. 
Deb, K. \& Goyal, M. (1996). A combined genetic adaptive search (GeneAS) for engineering design. Computer Science and Informatics, Vol. 26, No. 4, pp. 30-45.

Deb, K.; Pratap, A.; Agarwal, S. \& Meyarivan, T. (2002). A fast and elitist multiobjective genetic algorithm: NSGA-II. IEEE Transactions on Evolutionary Computation, Vol. 6, No. 2, pp. 182-197.

Dias, A.H.F. \& Vasconcelos, J.A. (2002). Multiobjective genetic algorithms applied to solve optimization problems. IEEE Transactions on Magnetics, Vol. 38, No. 2, pp. 11331136.

Ghomsheh, V.S.; Khanehsar, M.A. \& Teshnehlab, M. (2007). Improving the non-dominate sorting genetic algorithm for multi-objective optimization, Proceedings of Computational Intelligence and Security Workshops, pp. 89-92.

Goldberg, D.E. (1989). Genetic Algorithm in Search, Optimization, and Machine Leaning, Addison Wesley.

Horn, J.; Nafpliotis, N. \& Goldberg, D. (1994). A niched pareto genetic algorithm for multiobjective optimization, Proceedings of the First IEEE Conf. on Computational Intelligence, pp. 82-87.

Huband, S.; Hingston, P.; Barone, L. \& While, L. (2006). A review of multiobjective test problems and a scalable test problem toolkit. Evolutionary Computation, Vol. 10, No. 5, pp. 477-506.

Jaeggi, D.; Asselin-Miller, C.; Parks, G.; Kipouros, T.; Bell, T. \& Clarkson, J. (2004). Multiobjective Parallel Tabu Search. Lecture Notes in Computer Science, Vol. 3242, pp. 732741.

Knowles, J. \& Corne, D. (1999). The Pareto archived evolution strategy: A new baseline algorithm for multiobjective optimization, Proceedings of the 1999 Congress on Evolutionary Computation, pp. 98-105.

Lin, C.H. \& Chuang, C.C. (2007). A rough set penalty function for marriage selection in multiple-evaluation genetic algorithms. Lecture Notes in Computer Science, Vol. 4481, pp. 500-507.

Lin, C.H. \& He, J.D. (2007). Multiple-evaluation genetic algorithm for numerical optimization problems, Proceedings of the Computability in Europe 2007: Computation and Logic in the Real World, pp. 239-246.

Nebro, A.J.; Durillo, J.J.; Luna, F.; Dorronsoro, B. \& Alba, E. (2007). A cellular genetic algorithm for multiojective optimization. International Journal of Intelligent Systems, pp. 1-12.

Nebro, A.J.; Luna, F. \& Alba, E. (2005). New ideas in applying scatter search to multiobjective optimization, Proceedings of IEEE Conference on Evolutionary MultiCriterion Optimization Guanajuato, Mexico, pp. 443-458.

Okabe, T.; Jin, Y. \& Sendhoff, B. (2003). A critical survey of performance indices for multiobjective optimisation, Proceedings of the Congress on Evolutionary Computation, pp. 878-885.

Qi, R.; Qian, F.; Li, S. \& Wang, Z. (2006). Chaos-genetic algorithm for multiobjective optimization, Proceedings of the Sixth World Congress on Intelligent Control and Automation, pp. 1563-1566.

Ripon, K.S.N.; Kwong, S. \& Man, K.F. (2007). A real-coding jumping gene genetic algorithm (RJGGA) for multiobjective optimization. Information Sciences, Vol. 177, No. 2, pp. 632-654. 
Srinivas, N. \& Deb, K. (1994). Multiobjective optimization using nondominated sorting in genetic algorithms. Evolutionary Computation, Vol. 2, No. 3, pp. 221-248.

Zitzler, E. \& Thiele, L. (1999) Multiobjective evolutionary algorithms: A comparative case studyand the strength pareto approach. IEEE Transactions on Evolutionary Computation, Vol. 3, No. 4, pp. 257-271.

Zitzler, E.; Deb, K. \& Thiele, L. (2000). Comparison of multiobjective evolutionary algorithms: Empirical results. Evolutionary Computation, Vol. 8, No. 2, pp. 173-195. 


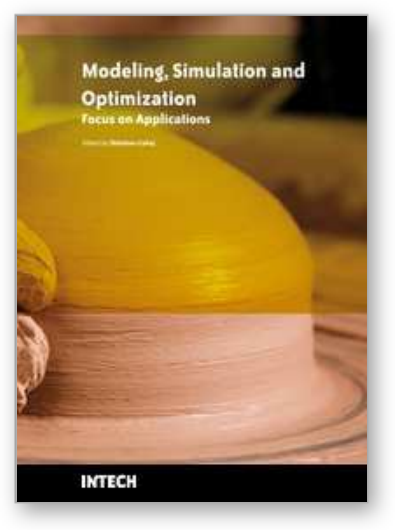

\author{
Modeling Simulation and Optimization - Focus on Applications \\ Edited by Shkelzen Cakaj
}

ISBN 978-953-307-055-1

Hard cover, 312 pages

Publisher InTech

Published online 01, March, 2010

Published in print edition March, 2010

The book presents a collection of chapters dealing with a wide selection of topics concerning different applications of modeling. It includes modeling, simulation and optimization applications in the areas of medical care systems, genetics, business, ethics and linguistics, applying very sophisticated methods. Algorithms, 3-D modeling, virtual reality, multi objective optimization, finite element methods, multi agent model simulation, system dynamics simulation, hierarchical Petri Net model and two level formalism modeling are tools and methods employed in these papers.

\title{
How to reference
}

In order to correctly reference this scholarly work, feel free to copy and paste the following:

Chih-Hao Lin and Pei-Ling Lin (2010). A New Non-dominated Sorting Genetic Algorithm for Multi-Objective Optimization, Modeling Simulation and Optimization - Focus on Applications, Shkelzen Cakaj (Ed.), ISBN: 978953-307-055-1, InTech, Available from: http://www.intechopen.com/books/modeling-simulation-andoptimization-focus-on-applications/a-new-non-dominated-sorting-genetic-algorithm-for-multi-objectiveoptimization

\section{INTECH}

open science | open minds

\section{InTech Europe}

University Campus STeP Ri

Slavka Krautzeka 83/A

51000 Rijeka, Croatia

Phone: +385 (51) 770447

Fax: +385 (51) 686166

www.intechopen.com

\section{InTech China}

Unit 405, Office Block, Hotel Equatorial Shanghai

No.65, Yan An Road (West), Shanghai, 200040, China

中国上海市延安西路65号上海国际贵都大饭店办公楼405单元

Phone: +86-21-62489820

Fax: $+86-21-62489821$ 
(C) 2010 The Author(s). Licensee IntechOpen. This chapter is distributed under the terms of the Creative Commons Attribution-NonCommercialShareAlike-3.0 License, which permits use, distribution and reproduction for non-commercial purposes, provided the original is properly cited and derivative works building on this content are distributed under the same license. 